\title{
Boundedness criterion for multilinear oscillatory integrals with rough kernels
}

\author{
by \\ Wengu Chen and Shanzhen Lu (Beijing)
}

\begin{abstract}
We study a multilinear oscillatory integral with rough kernel and establish a boundedness criterion.
\end{abstract}

1. Introduction. Let $\Omega$ be a homogeneous function of degree zero satisfying some size condition, for example, $\Omega \in L(\log L)^{\alpha}\left(S^{n-1}\right)$ for some $\alpha \geq 1$. This size condition is weaker than $\Omega \in \bigcup_{q>1} L^{q}\left(S^{n-1}\right)$. Under this assumption, we consider a multilinear oscillatory integral, which is related to Calderón commutators and defined by

$$
T^{A} f(x)=\text { p.v. } \int_{\mathbb{R}^{n}} e^{i P(x, y)} \frac{\Omega(x-y)}{|x-y|^{n+m-1}} P_{m}(A ; x, y) f(y) d y,
$$

where $n \geq 2, m$ is a positive integer, $P(x, y)$ is a real-valued polynomial defined on $\mathbb{R}^{n} \times \mathbb{R}^{n}$ and $P_{m}(A ; x, y)$ denotes the $m$ th order Taylor series remainder of $A$ at $x$ expanded about $y$, more precisely

$$
P_{m}(A ; x, y)=A(x)-\sum_{|\alpha|<m} \frac{1}{\alpha !} D^{\alpha} A(y)(x-y)^{\alpha} .
$$

Generally, it is impossible to derive $L^{2}$ boundedness of $T^{A}$ from the standard T1 theorem (see [2]) or nonstandard T1 theorem (see [3]), it is therefore necessary to establish some boundedness criterions. According to these criterions, the $L^{p}$ boundedness properties of these singular integrals are reduced to those of some truncated operators. The idea is hidden in the paper [5] of Ricci and Stein and put forward concretely by Lu and Zhang in [4].

Now, we introduce some notation. Let $(X, \mu)$ be a measure space and let $\Phi$ be a Young function. The Orlicz space $L_{\Phi}(X, \mu)$ consists of all $\mu$-measurable functions $f$ (modulo the a.e. equivalence relation) such that

2000 Mathematics Subject Classification: Primary 42B20.

The research was supported by NNSF of China (Nos. 19901021, 10371080 and 19131080) and Beijing Natural Science Foundation (No. 1013006). 


$$
\int_{X} \Phi(\varepsilon|f(x)|) d \mu(x)<\infty
$$

for some $\varepsilon>0$. The norm

$$
\|f\|_{\Phi}=\inf \left\{\lambda>0: \int_{X} \Phi\left(\frac{|f(x)|}{\lambda}\right) d \mu(x) \leq 1\right\}
$$

turns $L_{\Phi}$ into a Banach space. The space $L_{\Phi}$ can be endowed with another equivalent norm which is defined by

$$
\|f\|_{L_{\Phi}}=\inf \left\{\frac{1}{\varepsilon}\left(1+\int_{X} \Phi(\varepsilon|f|)\right): \int_{X} \Phi(\varepsilon|f|) d \mu<\infty\right\} .
$$

When $X=S^{n-1}$, the unit sphere of $\mathbb{R}^{n}, d \mu=d \sigma$, the element of Lebesgue measure on $S^{n-1}$ so that the measure of $S^{n-1}$ is 1 , and $\Phi(t)=t \log ^{\alpha}(2+t)$, $1 \leq \alpha<\infty$, we denote $L_{\Phi}$ by $L(\log L)^{\alpha}\left(S^{n-1}\right)$. We define the $\Phi$-average of a function $f$ over a cube $Q$ by

$$
\|f\|_{\Phi, Q}=\inf \left\{\lambda>0: \frac{1}{|Q|} \int_{Q} \Phi\left(\frac{|f(y)|}{\lambda}\right) d y \leq 1\right\} .
$$

Then the generalized Hölder inequality

$$
\frac{1}{|Q|} \int_{Q}|f(y) g(y)| d y \leq\|f\|_{\Phi, Q}\|g\|_{\bar{\Phi}, Q}
$$

holds, where $\bar{\Phi}$ is the complementary Young function associated to $\Phi$.

Definition 1. A real-valued polynomial $P(x, y)$ is called non-degenerate if there exist positive integers $k, l$ such that $P(x, y)=\sum_{|\alpha| \leq k,|\beta| \leq l} a_{\alpha \beta} x^{\alpha} y^{\beta}$ and $\sum_{|\alpha|=k,|\beta|=l}\left|a_{\alpha \beta}\right|>0$.

Definition 2. We will say that the non-degenerate polynomial $P(x, y)$ has property $\mathcal{P}$ if

$$
P(x+h, y+h)=P(x, y)+P_{0}(x, h)+P_{1}(y, h),
$$

where $P_{0}$ and $P_{1}$ are real polynomials.

The purpose of this paper is to establish the following boundedness criterion.

THEOREM 1. Let $\Omega \in L(\log L)^{2}\left(S^{n-1}\right)$ be homogeneous of degree zero. If $A$ has derivatives of order $m-1$ in $\mathrm{BMO}\left(\mathbb{R}^{n}\right)$, then for any $1<p<\infty$, the following two facts are equivalent:

(i) If $P(x, y)$ is a non-degenerate real-valued polynomial, then $T^{A}$ is bounded on $L^{p}$ with bound $C \sum_{|\alpha|=m-1}\left\|D^{\alpha} A\right\|_{\mathrm{BMO}}$, and the positive constant $C$ can be taken to be independent of the coefficients of the polynomial $P(x, y)$. 
(ii) The truncated operator

$$
S^{A} f(x)=\int_{|x-y|<1} \frac{\Omega(x-y)}{|x-y|^{n+m-1}} P_{m}(A ; x, y) f(y) d y
$$

is bounded on $L^{p}$ with bound $C \sum_{|\alpha|=m-1}\left\|D^{\alpha} A\right\|_{\mathrm{BMO}}$.

2. Proof of Theorem 1. To prove Theorem 1, we will use some lemmas.

LEMma 1 (see [1]). Let $b(x)$ be a function on $\mathbb{R}^{n}$ with mth order derivatives in $L^{s}\left(\mathbb{R}^{n}\right)$ for some $s$ with $n<s \leq \infty$. Then

$$
\left|P_{m}(b ; x, y)\right| \leq C_{m, n}|x-y|^{m} \sum_{|\alpha|=m}\left(\frac{1}{\left|I_{x}^{y}\right|} \int_{I_{x}^{y}}\left|D^{\alpha} b(z)\right|^{s} d z\right)^{1 / s},
$$

where $I_{x}^{y}$ is the cube centered at $x$, with sides parallel to the axes and whose diameter is $2 \sqrt{n}|x-y|$.

LEMma 2. Let $\Omega$ be a homogeneous function of degree zero and belong to $L \log L\left(S^{n-1}\right)$ and $A$ have derivatives of order $m-1$ in $\operatorname{BMO}\left(\mathbb{R}^{n}\right)$. Define

$$
S_{\Omega ; r}^{A} f(x)=r^{-(n+m-1)} \int_{|x-y|<r}\left|\Omega(x-y) P_{m}(A ; x, y) f(y)\right| d y .
$$

Then for any $1<p<\infty$,

$$
\left\|S_{\Omega ; r}^{A} f\right\|_{p} \leq C\left(1+\int_{S^{n-1}}|\Omega(x)| \log (2+|\Omega(x)|) d \sigma(x)\right)\|f\|_{p},
$$

where the constant $C>0$ is independent of $r$.

Proof. By dilation invariance, it suffices to consider the case $r=1$. By an almost orthogonality argument, we may assume that $f$ has support in a cube $Q$ with side length 1 . Without loss of generality, we may also assume $\sum_{|\alpha|=m-1}\left\|D^{\alpha} A\right\|_{\mathrm{BMO}}=1$. Define

$$
\Omega_{k}(x)=\Omega(x) \chi_{E_{k}}(x)
$$

with

$$
E_{k}(x)=\left\{x \in S^{n-1}: 2^{k-1} \leq|\Omega(x)|<2^{k}\right\}, \quad k \in \mathbb{Z},
$$

and for any $k \in \mathbb{Z}$, define an operator $T_{k}$ by

$$
T_{k} g(x)=\int_{|x-y| \leq 1}\left|\Omega_{k}(x-y)\right| g(y) d y .
$$

Denote by $T_{k}^{*}$ the dual operator of $T_{k}$. Then

$$
T_{k}^{*} g(x)=\int_{|x-y| \leq 1}\left|\Omega_{k}(y-x)\right| g(y) d y .
$$


We claim that there exists a positive constant $C=C(n, m)$ which is independent of $k$ such that for any $1 \leq p<\infty$,

$$
\left\|\left|T_{k}^{*} g\right|^{p}\right\|_{L(\log L)^{p}, Q} \leq C\left(2^{-|k|}+\int_{E_{k}}\left|\Omega_{k}(x)\right| \log \left(2+\left|\Omega_{k}(x)\right|\right) d \sigma(x)\right)^{p}\|g\|_{p}^{p}
$$

for any $g \in L^{p}$ with $\operatorname{supp} g \subset 100 n Q$. In fact, without loss of generality, we may assume that $\|g\|_{p}=1$. By the Young inequality, there exists some $C_{0}=C(n)>1$ such that

$$
\begin{aligned}
\left\|T_{k}^{*} g\right\|_{\infty} & \leq\left\|\Omega_{k}\right\|_{\infty}\|g\|_{1} \leq C_{0}\left\|\Omega_{k}\right\|_{\infty}, \\
\left\|T_{k}^{*} g\right\|_{p} & \leq\left\|\Omega_{k}\right\|_{1}\|g\|_{p}=\left\|\Omega_{k}\right\|_{1} .
\end{aligned}
$$

Write

$$
\begin{aligned}
\left\|\left|T_{k}^{*} g\right|^{p}\right\|_{L(\log L)^{p}, Q} & =\inf \left\{\lambda>0: \int_{Q} \frac{\left|T_{k}^{*} g(x)\right|^{p}}{\lambda} \log ^{p}\left(2+\frac{\left|T_{k}^{*} g(x)\right|^{p}}{\lambda}\right) d x \leq 1\right\} \\
& \leq \inf \left\{\lambda>0: \frac{\left\|\Omega_{k}\right\|_{1}^{p}}{\lambda} \log ^{p}\left(2+\frac{C_{0}^{p}\left\|\Omega_{k}\right\|_{\infty}^{p}}{\lambda}\right) \leq 1\right\} \\
& \leq C_{0}^{p}\left(\inf \left\{\lambda>0: \frac{2\left\|\Omega_{k}\right\|_{1}}{\lambda} \log \left(2+\frac{\left\|\Omega_{k}\right\|_{\infty}}{\lambda}\right) \leq 1\right\}\right)^{p} .
\end{aligned}
$$

Note that

$$
\frac{2\left\|\Omega_{k}\right\|_{1}}{2 \lambda} \log \left(2+\frac{\left\|\Omega_{k}\right\|_{\infty}}{2 \lambda}\right) \leq \int_{E_{k}} \frac{\left|\Omega_{k}(x)\right|}{\lambda} \log \left(2+\frac{\left|\Omega_{k}(x)\right|}{\lambda}\right) d \sigma(x) .
$$

Therefore,

$$
\left\|\left|T_{k}^{*} g\right|^{p}\right\|_{L(\log L)^{p}, Q} \leq C\left\|\Omega_{k}\right\|_{L \log L\left(S^{n-1}\right)}^{p} .
$$

For $k \geq 1$, since

$$
\int_{E_{k}} 2^{k}\left|\Omega_{k}(x)\right| \log \left(2+2^{k}\left|\Omega_{k}(x)\right|\right) d \sigma(x) \leq 2^{k} \cdot 2^{k} \cdot 2 k \cdot\left|E_{k}\right|<\infty,
$$

by the equivalence of the two norms, we have

$$
\begin{aligned}
\left\|\Omega_{k}\right\|_{L \log L\left(S^{n-1}\right)} & \leq C\left(\frac{1}{2^{k}}+\int_{E_{k}}\left|\Omega_{k}(x)\right| \log \left(2+2^{k}|\Omega(x)|\right) d \sigma(x)\right) \\
& \leq C\left(2^{-k}+\int_{E_{k}}\left|\Omega_{k}(x)\right| \log (2+|\Omega(x)|) d \sigma(x)\right) .
\end{aligned}
$$

For $k \leq 0$, since

$$
\int_{E_{k}} 2^{-k}\left|\Omega_{k}(x)\right| \log \left(2+2^{-k}\left|\Omega_{k}(x)\right|\right) d \sigma(x) \leq 2^{-k} \cdot 2^{k} \cdot \log 3 \cdot\left|E_{k}\right|<\infty,
$$


similarly, we have

$$
\begin{aligned}
\left\|\Omega_{k}\right\|_{L \log L\left(S^{n-1}\right)} & \leq C\left(\frac{1}{2^{-k}}+\int_{E_{k}}\left|\Omega_{k}(x)\right| \log \left(2+2^{-k}|\Omega(x)|\right) d \sigma(x)\right) \\
& \leq C\left(2^{k}+\int_{E_{k}}\left|\Omega_{k}(x)\right| d \sigma(x)\right) .
\end{aligned}
$$

Therefore,

$$
\left\|\left|T_{k}^{*} g\right|^{p}\right\|_{L(\log L)^{p}, Q} \leq C\left(2^{-|k|}+\int_{E_{k}}\left|\Omega_{k}(x)\right| \log \left(2+\left|\Omega_{k}(x)\right|\right) d \sigma(x)\right)^{p}\|g\|_{p}^{p} .
$$

Let $\phi \in C_{0}^{\infty}\left(\mathbb{R}^{n}\right), 0 \leq \phi \leq 1$, and let $\phi$ be identically one on $10 \sqrt{n} Q$ and vanish outside of $50 \sqrt{n} Q,\left\|\phi^{(\gamma)}\right\|_{\infty} \leq C_{\gamma}$ for all multi-indices $\gamma$. Let $x_{0}$ be a point on the boundary of $80 \sqrt{n} Q$. Define

$$
A_{\phi}(y)=P_{m-1}\left(A(\cdot)-\sum_{|\alpha|=m-1} \frac{1}{\alpha !} m_{\widetilde{Q}}\left(A^{(\alpha)}\right)(\cdot)^{\alpha} ; y, x_{0}\right) \phi(y),
$$

where $m_{Q}(f)=|Q|^{-1} \int_{Q} f$ and $\widetilde{Q}=100 n Q$. Note that for any multi-index $\beta$, $|\beta|<m-1$,

$$
\begin{aligned}
D^{\beta} A_{\phi}(y)= & \sum_{\beta=\mu+\nu} C_{\mu, \nu} P_{m-|\mu|-1}\left(D^{\mu}\left(A(\cdot)-\sum_{|\alpha|=m-1} \frac{1}{\alpha !} m_{\widetilde{Q}}\left(D^{\alpha} A\right)(\cdot)^{\alpha}\right) ; y, x_{0}\right) \\
& \times D^{\nu} \phi(y) .
\end{aligned}
$$

Since supp $\phi \subset 50 \sqrt{n} Q$, by Lemma 1 we have

$$
\left|D^{\beta} A_{\phi}(y)\right| \leq C \sum_{|\alpha|=m-1}\left(\frac{1}{\left|I_{x_{0}}^{y}\right|} \int_{I_{x_{0}}^{y}}\left|D^{\alpha} A(z)-m_{\widetilde{Q}}\left(D^{\alpha} A\right)\right|^{t} d z\right)^{1 / t} \leq C,
$$

where $t>n$. If $|\beta|=m-1$, then

$$
\begin{aligned}
D^{\beta} A_{\phi}(y) & =\sum_{\beta=\mu+\nu,|\mu|<m-1} C_{\mu, \nu} \\
& \times P_{m-1-|\mu|}\left(D^{\mu}\left(A(\cdot)-\sum_{|\alpha|=m-1} \frac{1}{\alpha !} m_{\widetilde{Q}}\left(D^{\alpha} A\right)(\cdot)^{\alpha}\right) ; y, x_{0}\right) D^{\nu} \phi(y) \\
& +\sum_{|\alpha|=m-1}\left(D^{\alpha} A(y)-m_{\widetilde{Q}}\left(D^{\alpha} A\right)\right) \phi(y) .
\end{aligned}
$$

Thus, it follows that

Since

$$
\left|D^{\beta} A_{\phi}(y)\right| \leq C\left(1+\sum_{|\alpha|=m-1}\left|D^{\alpha} A(y)-m_{\widetilde{Q}}\left(D^{\alpha} A\right)\right|\right) .
$$




$$
S_{\Omega ; 1}^{A} f(x) \leq \sum_{k=-\infty}^{\infty}\left(\left|A_{\phi}(x)\right| T_{k}(|f|)(x)+\sum_{|\alpha| \leq m-1} \frac{1}{\alpha !} T_{k}\left(\left|D^{\alpha} A_{\phi} f\right|\right)(x)\right),
$$

by the fact that the operator $T_{k}$ is bounded on $L^{p}$ together with the above inequalities, we obtain

$$
\left\|S_{\Omega ; 1}^{A} f\right\|_{p} \leq C \sum_{k=-\infty}^{\infty}\left(\left\|\Omega_{k}\right\|_{1}\|f\|_{p}+\sum_{|\alpha|=m-1}\left\|T_{k}\left(\left|D^{\alpha} A(\cdot)-m_{\widetilde{Q}}\left(D^{\alpha} A\right)\right||f|\right)\right\|_{p}\right) .
$$

For $|\alpha|=m-1$, by the generalized Hölder inequality and the fact that

we have

$$
\left\|D^{\alpha} A-m_{\widetilde{Q}}\left(D^{\alpha}\right)\right\|_{\exp L, \widetilde{Q}} \leq C\left\|D^{\alpha} A\right\|_{\mathrm{BMO}},
$$

$$
\begin{aligned}
& \left\|T_{k}\left(\left|D^{\alpha} A(\cdot)-m_{\widetilde{Q}}\left(D^{\alpha} A\right)\right||f|\right)\right\|_{p} \\
& =\sup _{\operatorname{supp} g \subset \widetilde{Q},\|g\|_{p^{\prime}}=1}\left|\int_{\widetilde{Q}} T_{k}\left(\left|D^{\alpha} A(\cdot)-m_{\widetilde{Q}}\left(D^{\alpha} A\right)\right||f|\right)(x) g(x) d x\right| \\
& =\sup _{\operatorname{supp} g \subset \widetilde{Q},\|g\|_{p^{\prime}}=1}\left|\int_{\widetilde{Q}}\right| D^{\alpha} A(x)-m_{\widetilde{Q}}\left(D^{\alpha} A\right)|| f(x)\left|T_{k}^{*} g(x) d x\right| \\
& \leq \sup _{\operatorname{supp} g \subset \widetilde{Q},\|g\|_{p^{\prime}}=1}\left(\int_{\widetilde{Q}}\left|D^{\alpha} A(x)-m_{\widetilde{Q}}\left(D^{\alpha} A\right)\right|^{p^{\prime}}\left|T_{k}^{*} g(x)\right|^{p^{\prime}} d x\right)^{1 / p^{\prime}}\|f\|_{p} \\
& \leq C \sup _{\operatorname{supp} g \subset \widetilde{Q},\|g\|_{p^{\prime}}=1}\left\|\left[D^{\alpha} A(\cdot)-m_{\widetilde{Q}}\left(D^{\alpha} A\right)\right]^{p^{\prime}}\right\|_{(\exp L)^{1 / p^{\prime}}, \widetilde{Q}}^{1 / p^{\prime}}\left\|\left|T_{k}^{*} g\right|^{p^{\prime}}\right\|_{L(\log L)^{p^{\prime}}, \widetilde{Q}}^{1 / p^{\prime}}\|f\|_{p} \\
& \leq C \sup _{\operatorname{supp} g \subset \widetilde{Q},\|g\|_{p^{\prime}}=1}\left\|\left|T_{k}^{*} g\right|^{p^{\prime}}\right\|_{L(\log L)^{p^{\prime}}, \widetilde{Q}}^{1 / p^{\prime}}\|f\|_{p} \\
& \leq C\left(2^{-|k|}+\int_{E_{k}}\left|\Omega_{k}(x)\right| \log \left(2+\left|\Omega_{k}(x)\right|\right) d \sigma(x)\right)\|f\|_{p} .
\end{aligned}
$$

Finally, we obtain

$$
\left\|S_{\Omega ; 1}^{A} f\right\|_{p} \leq C\left(1+\int_{S^{n-1}}|\Omega(x)| \log (2+|\Omega(x)|) d \sigma(x)\right)\|f\|_{p} .
$$

Lemma 3. Let $\Omega \in L \log L\left(S^{n-1}\right)$ be homogeneous of degree zero. Suppose that $A$ has derivatives of order $m-1$ in $\operatorname{BMO}\left(\mathbb{R}^{n}\right), b \in L^{\infty}\left(\mathbb{R}^{n} \times \mathbb{R}^{n}\right)$ and $1<p<\infty$. If the operator

$$
T f(x)=\text { p.v. } \int_{\mathbb{R}^{n}} \frac{\Omega(x-y)}{|x-y|^{n+m-1}} P_{m}(A ; x, y) b(x, y) f(y) d y
$$

is bounded on $L^{p}$ with bound $B \sum_{|\alpha|=m-1}\left\|D^{\alpha} A\right\|_{\mathrm{BMO}}$, then the truncated 
operator

$$
T_{1} f(x)=\int_{|x-y|<1} \frac{\Omega(x-y)}{|x-y|^{n+m-1}} P_{m}(A ; x, y) b(x, y) f(y) d y
$$

is bounded on $L^{p}$ with bound $C\left(B+\|b\|_{\infty}\right) \sum_{|\alpha|=m-1}\left\|D^{\alpha} A\right\|_{\mathrm{BMO}}$.

Proof. Without loss of generality, we may assume that

$$
\sum_{|\alpha|=m-1}\left\|D^{\alpha} A\right\|_{\mathrm{BMO}}=1 \text {. }
$$

For each fixed $h \in \mathbb{R}^{n}$, we split $f=f_{1}+f_{2}+f_{3}$, where

$$
f_{1}(y)=f(y) \chi_{\{|y-h|<1 / 2\}}(y), \quad f_{2}(y)=f(y) \chi_{\{1 / 2 \leq|y-h|<5 / 4\}}(y) .
$$

It is easy to verify that if $|x-h|<1 / 4$, then

$$
T_{1} f_{1}(x)=\int_{\mathbb{R}^{n}} \frac{\Omega(x-y)}{|x-y|^{n+m-1}} P_{m}(A ; x, y) b(x, y) f_{1}(y) d y .
$$

Thus

$$
\int_{|x-h|<1 / 4}\left|T_{1} f_{1}(x)\right|^{p} d x \leq B^{p}\left\|f_{1}\right\|_{p}^{p} .
$$

If $|x-h|<1 / 4$ and $1 / 2 \leq|y-h|<5 / 4$, then $1 / 4<|x-y|<3 / 2$. So we see that for $|x-h|<1 / 4$,

$$
\begin{aligned}
\left|T_{1} f_{2}(x)\right| & \leq\|b\|_{\infty} \int_{1 / 4<|x-y|<3 / 2}\left|\frac{\Omega(x-y)}{|x-y|^{n+m-1}} P_{m}(A ; x, y) f_{2}(y)\right| d y \\
& \leq C\|b\|_{\infty} S_{\Omega ; 3 / 2}^{A} f_{2}(x) .
\end{aligned}
$$

Lemma 2 now tells us that

$$
\int_{|x-h|<1 / 4}\left|T_{1} f_{2}(x)\right|^{p} d x \leq C\|b\|_{\infty}^{p}\left\|f_{2}\right\|_{p}^{p} .
$$

Obviously, we have $T_{1} f_{3}=0$ for $|x-h|<1 / 4$. Combining the above inequalities leads to

$$
\int_{|x-h|<1 / 4}\left|T_{1} f(x)\right|^{p} d x \leq C\left(B^{p}+\|b\|_{\infty}^{p}\right) \int_{|y-h|<2}|f(y)|^{p} d y .
$$

Integrating the last inequality with respect to $h$ gives

$$
\left\|T_{1} f\right\|_{p} \leq C\left(B+\|b\|_{\infty}\right)\|f\|_{p} .
$$

This completes the proof of Lemma 3 .

Proof of Theorem 1. We only deal with the case that

$$
\sum_{|\alpha|=m-1}\left\|D^{\alpha} A\right\|_{\mathrm{BMO}}=1 \text {. }
$$


First we show that (ii) implies (i). Let $k$ and $l$ be two positive integers, and $P(x, y)$ be a non-degenerate real-valued polynomial with degree $k$ in $x$ and $l$ in $y$. Write

$$
P(x, y)=\sum_{|\alpha| \leq k,|\beta| \leq l} a_{\alpha \beta} x^{\alpha} y^{\beta} .
$$

By dilation invariance, we may assume that $\sum_{|\alpha|=k,|\beta|=l}\left|a_{\alpha \beta}\right|=1$. Decompose

$$
\begin{aligned}
T^{A} f(x)= & \int_{|x-y|<1} e^{i P(x, y)} \frac{\Omega(x-y)}{|x-y|^{n+m-1}} P_{m}(A ; x, y) f(y) d y \\
& +\sum_{d=1}^{\infty} \int_{2^{d-1} \leq|x-y|<2^{d}} e^{i P(x, y)} \frac{\Omega(x-y)}{|x-y|^{n+m-1}} P_{m}(A ; x, y) f(y) d y \\
= & T_{0}^{A} f(x)+\sum_{d=1}^{\infty} T_{d}^{A} f(x) .
\end{aligned}
$$

We first consider $T_{d}^{A}, d \geq 1$. Split

$$
T_{d}^{A} f(x)=\sum_{l=0}^{\infty} T_{\Omega_{l}, d}^{A} f(x),
$$

where

$$
\begin{aligned}
T_{\Omega_{l}, d}^{A} f(x) & =\int_{2^{d-1} \leq|x-y|<2^{d}} e^{i P(x, y)} \frac{\Omega_{l}(x-y)}{|x-y|^{n+m-1}} P_{m}(A ; x, y) f(y) d y, \\
\Omega_{l}\left(x^{\prime}\right) & =\Omega\left(x^{\prime}\right) \chi_{E_{l}}\left(x^{\prime}\right)
\end{aligned}
$$

with

$$
\begin{aligned}
& E_{0}=\left\{x^{\prime} \in S^{n-1}:\left|\Omega\left(x^{\prime}\right)\right|<1\right\}, \\
& E_{l}=\left\{x^{\prime} \in S^{n-1}: 2^{l-1} \leq\left|\Omega\left(x^{\prime}\right)\right|<2^{l}\right\}, \quad l \in \mathbb{N} .
\end{aligned}
$$

If we can prove that for some $\delta>0$,

$$
\left\|T_{\Omega_{l}, d}^{A} f\right\|_{p} \leq C 2^{-\delta d}\left\|\Omega_{l}\right\|_{\infty}\|f\|_{p}
$$

and

$$
\left\|T_{\Omega_{l}, d}^{A} f\right\|_{p} \leq C\left(2^{-l}+\int_{E_{l}}\left|\Omega_{l}(x)\right| \log \left(2+\left|\Omega_{l}(x)\right|\right) d \sigma(x)\right)\|f\|_{p},
$$

then, for a suitably chosen integer $M>\delta^{-1}$, we have

$$
\begin{aligned}
\left\|\sum_{d=1}^{\infty} T_{d}^{A} f\right\|_{p} \leq \sum_{d=1}^{\infty} \sum_{l=0}^{\infty}\left\|T_{\Omega_{l}, d}^{A} f\right\|_{p} \\
=\sum_{d=1}^{\infty}\left\|T_{\Omega_{0}, d}^{A} f\right\|_{p}+\sum_{l=1}^{\infty} \sum_{1 \leq d<M l}\left\|T_{\Omega_{l}, d}^{A} f\right\|_{p}+\sum_{l=1}^{\infty} \sum_{d \geq M l}\left\|T_{\Omega_{l}, d}^{A} f\right\|_{p}
\end{aligned}
$$




$$
\begin{aligned}
& \leq C\left\|\Omega_{0}\right\|_{\infty}\|f\|_{p}+\sum_{l=1}^{\infty} M l\left(2^{-l}+2^{l} l\left|E_{l}\right|\right)\|f\|_{p}+\sum_{l=1}^{\infty} \sum_{d \geq M l} 2^{-\delta d_{2}} 2^{l}\|f\|_{p} \\
& \leq C\left(1+\int_{S^{n-1}}|\Omega(x)| \log ^{2}(2+|\Omega(x)|) d \sigma(x)\right)\|f\|_{p} .
\end{aligned}
$$

Inequality (4) can be seen from the proof of Lemma 2. To prove (3), define

$$
\widetilde{T}_{\Omega_{l}, d}^{A} f(x)=\int_{1<|x-y| \leq 2} e^{i P\left(2^{d-1} x, 2^{d-1} y\right)} \frac{\Omega_{l}(x-y)}{|x-y|^{n+m-1}} P_{m}(A ; x, y) f(y) d y .
$$

By dilation invariance, it is enough to prove that

$$
\left\|\widetilde{T}_{\Omega_{l}, d}^{A} f\right\|_{p} \leq C 2^{-\delta d}\left\|\Omega_{l}\right\|_{\infty}\|f\|_{p} .
$$

By an almost orthogonality argument, we may assume that $f$ has support in a cube $Q$ with side length 1 . Let

$$
A_{\phi}(y)=P_{m-1}\left(A(\cdot)-\sum_{|\alpha|=m-1} \frac{1}{\alpha !} m_{\widetilde{Q}}\left(D^{\alpha} A\right)(\cdot)^{\alpha} ; y, x_{0}\right) \phi(y),
$$

where $\phi$ is as in the proof of Lemma 2. For a multi-index $\alpha$, define

$$
\widetilde{T}_{\Omega_{l}, d}^{\alpha} f(x)=\int_{1<|x-y| \leq 2} e^{i P\left(2^{d-1} x, 2^{d-1} y\right)} \frac{\Omega_{l}(x-y)}{|x-y|^{n+m-1}}(x-y)^{\alpha} f(y) d y .
$$

It is easy to see that

$$
\begin{aligned}
\widetilde{T}_{\Omega_{l}, d}^{A} f(x)= & \int_{1<|x-y| \leq 2} e^{i P\left(2^{d-1} x, 2^{d-1} y\right)} \frac{\Omega_{l}(x-y)}{|x-y|^{n+m-1}} P_{m}\left(A_{\phi} ; x, y\right) f(y) d y \\
= & A_{\phi}(x) \widetilde{T}_{\Omega_{l}, d}^{0} f(x)-\sum_{|\alpha|<m-1} \frac{1}{\alpha !} \widetilde{T}_{\Omega_{l}, d}^{\alpha}\left(D^{\alpha} A_{\phi} f\right)(x) \\
& -\sum_{|\alpha|=m-1} \frac{1}{\alpha !} \widetilde{T}_{\Omega_{l}, d}^{\alpha}\left(D^{\alpha} A_{\phi} f\right)(x) \\
= & I+I I+I I I .
\end{aligned}
$$

Before we estimate these terms, we define

$$
T_{\Omega_{l}, d}^{\alpha} f(x)=\int_{2^{d-1} \leq|x-y|<2^{d}} e^{i P(x, y)} \frac{\Omega_{l}(x-y)}{|x-y|^{n+m-1}}(x-y)^{\alpha} f(y) d y .
$$

Recall that $P(x, y)=\sum_{|\alpha| \leq k,|\beta| \leq l} a_{\alpha \beta} x^{\alpha} y^{\beta}$ and $\sum_{|\alpha|=k,|\beta|=l}\left|a_{\alpha \beta}\right|=1$. By a similar argument to that in [4], we can prove

Lemma 4. There exists a $\delta>0$ such that

$$
\left\|T_{\Omega_{l}, d}^{\alpha} f\right\|_{p} \leq C 2^{-d(\delta+m-1-|\alpha|)}\left\|\Omega_{l}\right\|_{\infty}\|f\|_{p},
$$


and $C>0$ can be taken to be independent of $d$ and the coefficients of $P(x, y)$.

We return to the estimates of $I, I I$ and $I I I$. Note that for a multi-index $\beta$ with $|\beta|<m-1$,

$$
\left\|D^{\beta} A_{\phi}\right\|_{\infty} \leq C .
$$

Thus, it follows from Lemma 4 that

$$
\|I\|_{p} \leq\left\|A_{\phi}\right\|_{\infty}\left\|\widetilde{T}_{\Omega_{l}, d}^{0} f\right\|_{p} \leq C 2^{-\delta d}\|f\|_{p} .
$$

Similarly, we have

$$
\|I I\|_{p} \leq C 2^{-\delta d}\|f\|_{p}
$$

It remains to estimate the third term III. Note that for any $0<\gamma<n$,

$$
\begin{aligned}
\left|\widetilde{T}_{\Omega_{l}, d}^{\alpha} f(x)\right| & \leq C \int_{1<|x-y| \leq 2}\left|\Omega_{l}(x-y) f(y)\right| d y \\
& \leq C_{\gamma}\left\|\Omega_{l}\right\|_{\infty} \int_{1<|x-y| \leq 2} \frac{|f(y)|}{|x-y|^{n-\gamma}} d y \\
& \leq C_{\gamma}\left\|\Omega_{l}\right\|_{\infty} I_{\gamma}(|f|)(x),
\end{aligned}
$$

where $I_{\gamma}$ denotes the usual fractional integral of order $\gamma$. For any $\sigma>0$ such that $1 /(p+\sigma)=1 / p-\gamma / n$, by the Hardy-Littlewood-Sobolev theorem [6], we get

$$
\left\|\widetilde{T}_{\Omega_{l}, d}^{\alpha} f\right\|_{p+\sigma} \leq C\left\|\Omega_{l}\right\|_{\infty}\|f\|_{p} .
$$

Lemma 4, inequality (7), and interpolation give

$$
\left\|\widetilde{T}_{\Omega_{l}, d}^{\alpha} f\right\|_{p} \leq C 2^{-\delta^{\prime} d}\left\|\Omega_{l}\right\|_{\infty}\|f\|_{p-\sigma},
$$

where $\delta^{\prime}$ is another positive constant and $0<\sigma<\sigma_{p}$. On the other hand, if $|\beta|=m-1$, then

$$
\left|D^{\beta} A_{\phi}(y)\right| \leq C\left(1+\sum_{|\alpha|=m-1}\left|D^{\alpha} A(y)-m_{\widetilde{Q}}\left(D^{\alpha} A\right)\right|\right),
$$

and this shows that for any $t>1$,

$$
\left\|D^{\beta} A_{\phi}\right\|_{t} \leq C_{t}
$$

By inequalities (8) and (9), we obtain

$$
\begin{aligned}
\|I I I\|_{p} & \leq C 2^{-\delta^{\prime} d} \sum_{|\alpha|=m-1}\left\|D^{\alpha} A_{\phi} f\right\|_{p-\sigma} \leq C 2^{-\delta^{\prime} d} \sum_{|\alpha|=m-1}\left\|D^{\alpha} A_{\phi}\right\|_{t}\|f\|_{p} \\
& \leq C 2^{-\delta^{\prime} d}\|f\|_{p},
\end{aligned}
$$

where we choose $0<\sigma<\sigma_{p}$ and $1<t<\infty$ such that $1 / p+1 / t=1 /(p-\sigma)$. All the above estimates imply that inequality (3) is true.

We turn our attention to the operator $T_{0}^{A}$. The estimate for this operator comes from the following lemma. 
Lemma 5. Let $\Omega \in L \log L\left(S^{n-1}\right)$ be homogeneous of degree zero and $A$ have derivatives of order $m-1$ in $\mathrm{BMO}\left(\mathbb{R}^{n}\right)$. Suppose that condition (ii) in Theorem 1 holds. Then for any real-valued polynomial $\widetilde{P}(x, y)$, the operator

$$
U^{A} f(x)=\int_{|x-y|<1} e^{i \widetilde{P}(x, y)} \frac{\Omega(x-y)}{|x-y|^{n+m-1}} P_{m}(A ; x, y) f(y) d y
$$

satisfies

$$
\left\|U^{A} f\right\|_{p} \leq C\|f\|_{p} .
$$

Proof. We shall argue by a double induction on the degree of the polynomial in $x$ and $y$. If the polynomial $\widetilde{P}(x, y)$ depends only on $x$ or $y$, it is obvious that condition (ii) implies (10). Let $u$ and $v$ be two positive integers and suppose the polynomial has degree $u$ in $x$ and $v$ in $y$. We assume that (10) holds for all polynomials which are sums of monomials of degree less than $u$ in $x$ times monomials of any degree in $y$, together with monomials which are of degree $u$ in $x$ times monomials which are of degree less than $v$ in $y$. Write $\widetilde{P}(x, y)$ as

$$
\widetilde{P}(x, y)=\sum_{|\alpha|=u,|\beta|=v} b_{\alpha \beta} x^{\alpha} y^{\beta}+P_{0}(x, y),
$$

where $P_{0}(x, y)$ satisfies the inductive assumption. Without loss of generality, we may assume that $\sum_{|\alpha|=u,|\beta|=v}\left|b_{\alpha \beta}\right| \leq 1$. Rewrite

$$
\widetilde{P}(x, y)=\sum_{|\alpha|=u,|\beta|=v} b_{\alpha \beta}\left(x^{\alpha} y^{\beta}-y^{\alpha+\beta}\right)+\widetilde{P}_{0}(x, y),
$$

where $\widetilde{P}_{0}(x, y)$ satisfies the inductive assumption. It follows that

$$
\begin{aligned}
U^{A} f(x)= & \int_{|x-y|<1} e^{i \widetilde{P}_{0}(x, y)} \frac{\Omega(x-y)}{|x-y|^{n+m-1}} P_{m}(A ; x, y) f(y) d y \\
& +\int_{|x-y|<1}\left(e^{i \widetilde{P}(x, y)}-e^{i \widetilde{P}_{0}(x, y)}\right) \frac{\Omega(x-y)}{|x-y|^{n+m-1}} P_{m}(A ; x, y) f(y) d y \\
= & U_{1}^{A} f(x)+U_{2}^{A} f(x) .
\end{aligned}
$$

Our inductive assumption now states that

$$
\left\|U_{1}^{A} f\right\|_{p} \leq C\|f\|_{p} .
$$

Set $\widetilde{f}(y)=f(y) \chi_{\{|y| \leq 2\}}$. It is easy to see $U_{2}^{A} f(x)=U_{2}^{A} \widetilde{f}(x)$ for $|x| \leq 1$. Thus, when $|x| \leq 1$, 


$$
\begin{aligned}
\left|U_{2}^{A} f(x)\right| & \leq C \int_{|x-y|<1}\left|\frac{\Omega(x-y)}{|x-y|^{n+m-2}} P_{m}(A ; x, y) \widetilde{f}(y)\right| d y \\
& \leq C \sum_{d=-\infty}^{0} 2^{d} 2^{-d(n+m-1)} \int_{2^{d-1} \leq|x-y|<2^{d}}\left|\Omega(x-y) P_{m}(A ; x, y) \widetilde{f}(y)\right| d y \\
& \leq C \sum_{d=-\infty}^{0} 2^{d} S_{\Omega ; 2^{d}}^{A} \widetilde{f}(x) .
\end{aligned}
$$

By Lemma 2, we get

$$
\begin{aligned}
& \left(\int_{|x| \leq 1}\left|U_{2}^{A} f\right|^{p} d x\right)^{1 / p} \leq C \sum_{d=-\infty}^{0} 2^{d}\left\|S_{\Omega ; 2^{d}}^{A} \tilde{f}\right\|_{p} \\
& \quad \leq C \sum_{d=-\infty}^{0} 2^{d}\left(1+\int_{S^{n-1}}|\Omega(x)| \log (2+|\Omega(x)|) d \sigma(x)\right)\left(\int_{|y| \leq 2}|f(y)|^{p} d y\right)^{1 / p} \\
& \quad \leq C\left(1+\int_{S^{n-1}}|\Omega(x)| \log (2+|\Omega(x)|) d \sigma(x)\right)\left(\int_{|y| \leq 2}|f(y)|^{p} d y\right)^{1 / p}
\end{aligned}
$$

from which the same argument as that in $[5$, p. 189] shows that the inequality

$$
\left(\int_{|x-h| \leq 1}\left|U_{2}^{A} f\right|^{p} d x\right)^{1 / p} \leq C\left(\int_{|y-h| \leq 2}|f(y)|^{p} d y\right)^{1 / p}
$$

holds for all $h \in \mathbb{R}^{n}$ and $C>0$ is independent of $h$. Integrating the last inequality with respect to $h$ and using Hölder's inequality, we finally obtain

$$
\left\|U_{2}^{A} f\right\|_{p} \leq C\|f\|_{p}
$$

Now we return to the proof of Theorem 1 and show that (i) implies (ii). To do this, we need to use Definition 2. We choose $Q(x, y)$ such that $Q(x, y)$ has property $\mathcal{P}$ and decompose

$$
\begin{aligned}
T^{A} f(x)= & \int_{|x-y|<1} e^{i Q(x, y)} \frac{\Omega(x-y)}{|x-y|^{n+m-1}} P_{m}(A ; x, y) f(y) d y \\
& +\int_{|x-y| \geq 1} e^{i Q(x, y)} \frac{\Omega(x-y)}{|x-y|^{n+m-1}} P_{m}(A ; x, y) f(y) d y \\
= & T_{0}^{A} f(x)+T_{\infty}^{A} f(x) .
\end{aligned}
$$

By Lemma $3, T_{0}^{A}$ is bounded on $L^{p}$. The same argument as in the proof of Lemma 3 tells us that 


$$
\left(\int_{|x-h|<1}\left|T_{0}^{A} f(x)\right|^{p} d x\right)^{1 / p} \leq C\left(\int_{|y-h|<2}|f(y)|^{p} d y\right)^{1 / p},
$$

where $C$ is independent of $h$. Since $Q(x, y)$ has property $\mathcal{P}$, we have

$$
Q(x, y)=Q(x-h, y-h)+P_{0}(x, h)+P_{1}(y, h),
$$

where $P_{0}, P_{1}$ are real polynomials. When $|x-h|<1$, it follows that

$$
\begin{aligned}
S^{A} f(x)= & \int_{|x-y|<1} \frac{\Omega(x-y)}{|x-y|^{n+m-1}} P_{m}(A ; x, y) f(y) \chi_{B(h, 2)}(y) d y \\
= & e^{-i P_{0}(x, h)} \int_{|x-y|<1} e^{i Q(x, y)} \frac{\Omega(x-y)}{|x-y|^{n+m-1}} P_{m}(A ; x, y) \\
& \times e^{-i Q(x-h, y-h)} e^{-i P_{1}(y, h)} f(y) \chi_{B(h, 2)}(y) d y .
\end{aligned}
$$

Observe that the Taylor expression of $e^{-i Q(x-h, y-h)}$ is

$$
\begin{aligned}
e^{-i Q(x-h, y-h)} & =\sum_{m=0}^{\infty} \frac{i^{m}}{m !}\left(\sum_{\alpha, \beta} a_{\alpha \beta}(x-h)^{\alpha}(y-h)^{\beta}\right)^{m} \\
& =\sum_{m=0}^{\infty} \frac{i^{m}}{m !} \sum_{u, v} a_{m, u, v}(x-h)^{u}(y-h)^{v} .
\end{aligned}
$$

If we set $a=(1,1, \ldots, 1) \in \mathbb{R}^{n}$ and $b=(2,2, \ldots, 2) \in \mathbb{R}^{n}$, then we have

$$
\begin{aligned}
\left(\int_{|x-h|<1} \mid S^{A}\right. & \left.\left.f(x)\right|^{p} d x\right)^{1 / p} \\
\leq & \sum_{m=0}^{\infty} \frac{1}{m !} \sum_{u, v}\left|a_{m, u, v}\right|\left(\int_{|x-h|<1}\left|(x-h)^{u}\right|^{p}\right. \\
& \left.\times\left|T_{0}^{A}\left(e^{-i P_{1}(\cdot, h)} f(\cdot) \chi_{B(h, 2)}(\cdot)(\cdot-h)^{v}\right)(x)\right|^{p} d x\right)^{1 / p} \\
\leq & \sum_{m=0}^{\infty} \frac{1}{m !} \sum_{u, v}\left|a_{m, u, v}\right| a^{u}\left(\int_{|y-h|<2}|f(y)|^{p}\left|(y-h)^{v}\right|^{p} d y\right)^{1 / p} \\
\leq & \sum_{m=0}^{\infty} \frac{1}{m !} \sum_{u, v}\left|a_{m, u, v}\right| a^{u} b^{v}\left(\int_{|y-h|<2}|f(y)|^{p} d y\right)^{1 / p} \\
= & \sum_{m=0}^{\infty} \frac{1}{m !}\left(\sum_{\alpha, \beta}\left|a_{\alpha \beta}\right| a^{\alpha} b^{\beta}\right)^{m}\left(\int_{|y-h|<2}|f(y)|^{p} d y\right)^{1 / p} \\
= & \exp \left\{\sum_{\alpha, \beta}\left|a_{\alpha \beta}\right| a^{\alpha} b^{\beta}\right\}\left(\int_{|y-h|<2}|f(y)|^{p} d y\right)^{1 / p} .
\end{aligned}
$$


Hence,

$$
\left\|S^{A} f\right\|_{p} \leq C\|f\|_{p} .
$$

This completes the proof of Theorem 1 .

Acknowledgements. The authors would like to express their deep thanks to the referee for his several valuable remarks and suggestions. Moreover, the first author would like to thank Professor Guoen $\mathrm{Hu}$ for his useful suggestions on this topic.

\section{References}

[1] J. Cohen and J. Gosselin, A BMO estimate for multilinear singular integrals, Illinois J. Math. 30 (1986), 445-464.

[2] G. David and J.-L. Journé, A boundedness criterion for generalized Calderón-Zygmund operators, Ann. of Math. 120 (1984), 371-397.

[3] S. Hofmann, On certain nonstandard Calderón-Zygmund operators, Studia Math. 109 (1994), 105-131.

[4] S. Z. Lu and Y. Zhang, Criterion on $L^{p}$-boundedness for a class of oscillatory singular integrals with rough kernels, Rev. Mat. Iberoamericana 8 (1992), 201-219.

[5] F. Ricci and E. M. Stein, Harmonic analysis on nilpotent groups and singular integrals I. Oscillatory integrals, J. Funct. Anal. 73 (1987), 179-194.

[6] E. M. Stein, Singular Integrals and Differentiability Properties of Functions, Princeton Univ. Press, Princeton, NJ, 1970.

Wengu Chen

Institute of Applied Physics

and Computational Mathematics

P.O. Box 8009

Beijing 100088, People's Republic of China

E-mail: chenwg@iapcm.ac.cn
Shanzhen Lu

Department of Mathematics Beijing Normal University

Beijing 100875, People's Republic of China E-mail: lusz@bnu.edu.cn

Received July 24, 2000

Revised version July 5, 2004 